\title{
General Collective Intelligence as a System for Facilitating Parallel and Distributed Computing
}

\author{
Andy E. Williams, Nobeah Foundation, Nairobi, Kenya
}

\begin{abstract}
A General Collective Intelligence or GCI is a hypothetical platform able to self-organize individuals or intelligent agents working on behalf of those individuals, into potentially massive networks of cooperation on a self-sustaining basis, where those networks might exponentially increase the complexity and duration of reasoning the group is able to execute. When applied to the computing processes that might be executed by each individual, the algorithm represented by GCI creates the potential to take any computing process that has been abstracted into a logical model (a model in which each component of logic might be implemented in a separate functional component), and to automatically search for and apply patterns of solution by which it might be possible to increase outcomes. Such patterns might involve distributing processing across multiple instances of those functional components, and scaling processing by increasing the instances of those functional components in series and in parallel, as well as ensuring this effort has more value than cost to performance so it is possible to sustain the effort at doing so, until computing outcomes are scaled as necessary to achieve a given target, or until returns from scaling diminish to the point that further investment in scaling is unproductive. This paper explores how such processes of cooperation might include all forms of parallel and distributed activity, how that cooperative activity might be abstracted into a logical model, and how GCI might facilitate parallel and distributed computing through that model.
\end{abstract}

\section{Keywords:}

General Collective Intelligence, parallel computing, distributed computing

\section{Introduction}

A General Collective Intelligence or GCI [1] is a hypothetical platform able to self-organize individuals or intelligent agents working on behalf of those individuals, into potentially massive networks of cooperation on a self-sustaining basis, where those networks might exponentially increase the general problem-solving ability (collective intelligence) of the group. This entails facilitating such cooperation between the computing tools each of those users might employ. Facilitating this cooperation for all possible programs requires either a complete representation of human meaning (a complete semantic model) of programs (a complete model of what a program might do and therefore a complete semantic model of program logic), or a model that can be extended to include any new computing functionality and any new logic. A complete model capable of representing any logic and a model that might be extended to include any possible new logic might be one and the same.

The technique of Human-Centric Functional Modeling that has been used to create a common model of information for GCI to manipulate, defines a means of representing the behavior of any system in terms of "functional state spaces" [2]. In these functional state spaces the set of human observable processes or functions which the system might use to transition from one functional state to another, form a graph. In the network of this graph, these functional states are nodes, and the processes by which one functional state might transition to another are edges connecting those nodes. Systems described this way then move through this functional state space as their processes are executed.

One example of a system modeled this way is artificial or human cognition [3], which can be represented as using reasoning processes to navigate from one concept (one functional state) to another concept (another functional state). This space of concepts or "conceptual space" serves as the 
functional state space the cognition moves through (navigates) as it thinks. All these reasoning processes consist of either type 1 (intuitive) reasoning that is represented as a direct path between two concepts in conceptual space, or type 2 (rational methodical) reasoning which is represented as a path through intermediate concepts constituting a chain of logic [4].
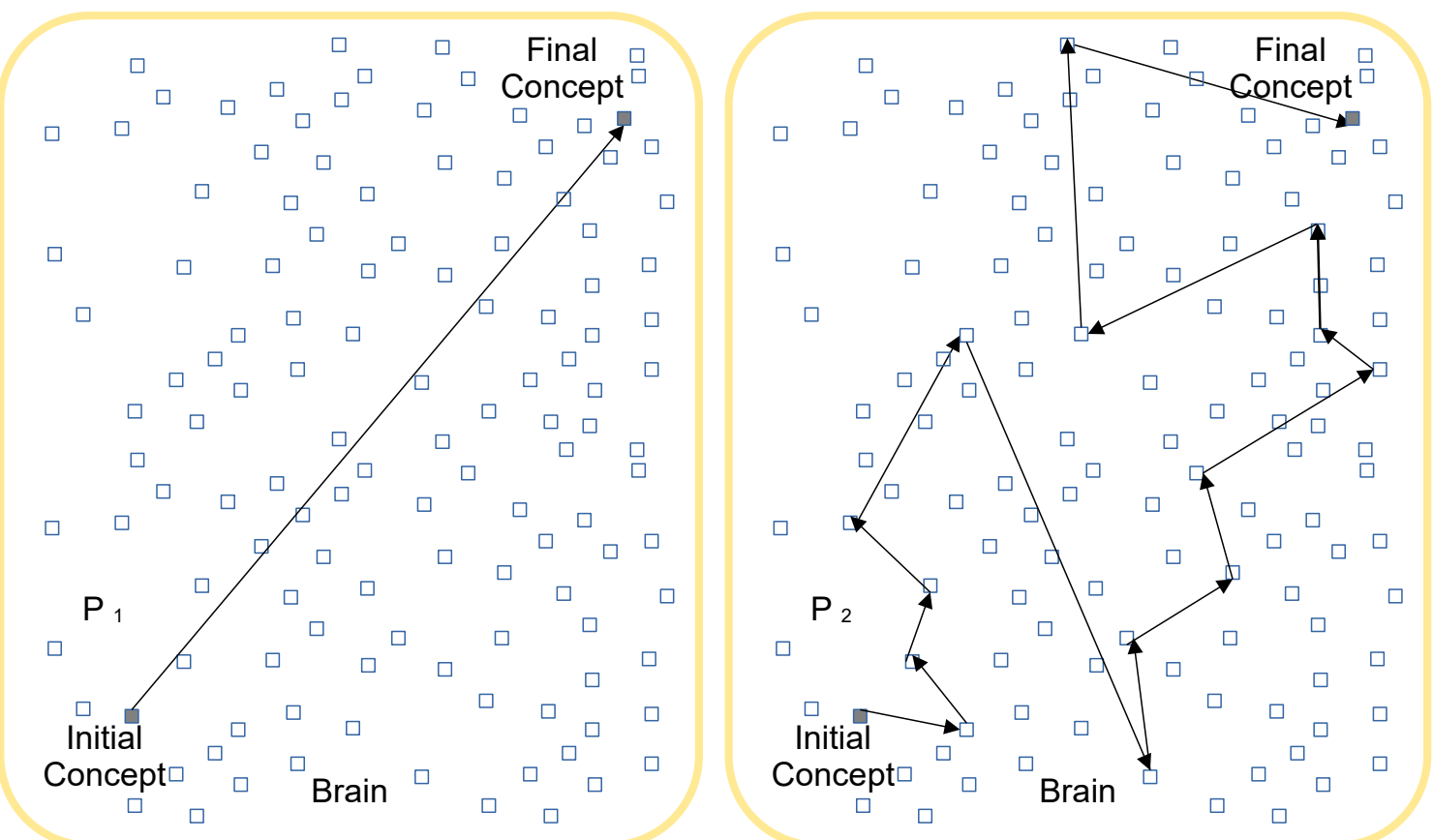

Figure 1: Type 1 and type 2 reasoning. Left: An intuitive (type 1) reasoning process in the brain. A simple intuitive reasoning process reaches a short distance in conceptual space. A complex intuitive reasoning process reaches a great distance in the conceptual space. Right: A complex rational methodical (type 2) reasoning process in the brain again reaches a great distance. The capacity to execute more complex reasoning is hypothesized to increase outcomes of problem-solving.

Assume that all execution of reasoning in the brain is distributed across multiple functional components, each of which plays a part in the reasoning process. This collaboration between functional components might be represented by a swim lane diagram of the reasoning process as in figure 2 . 


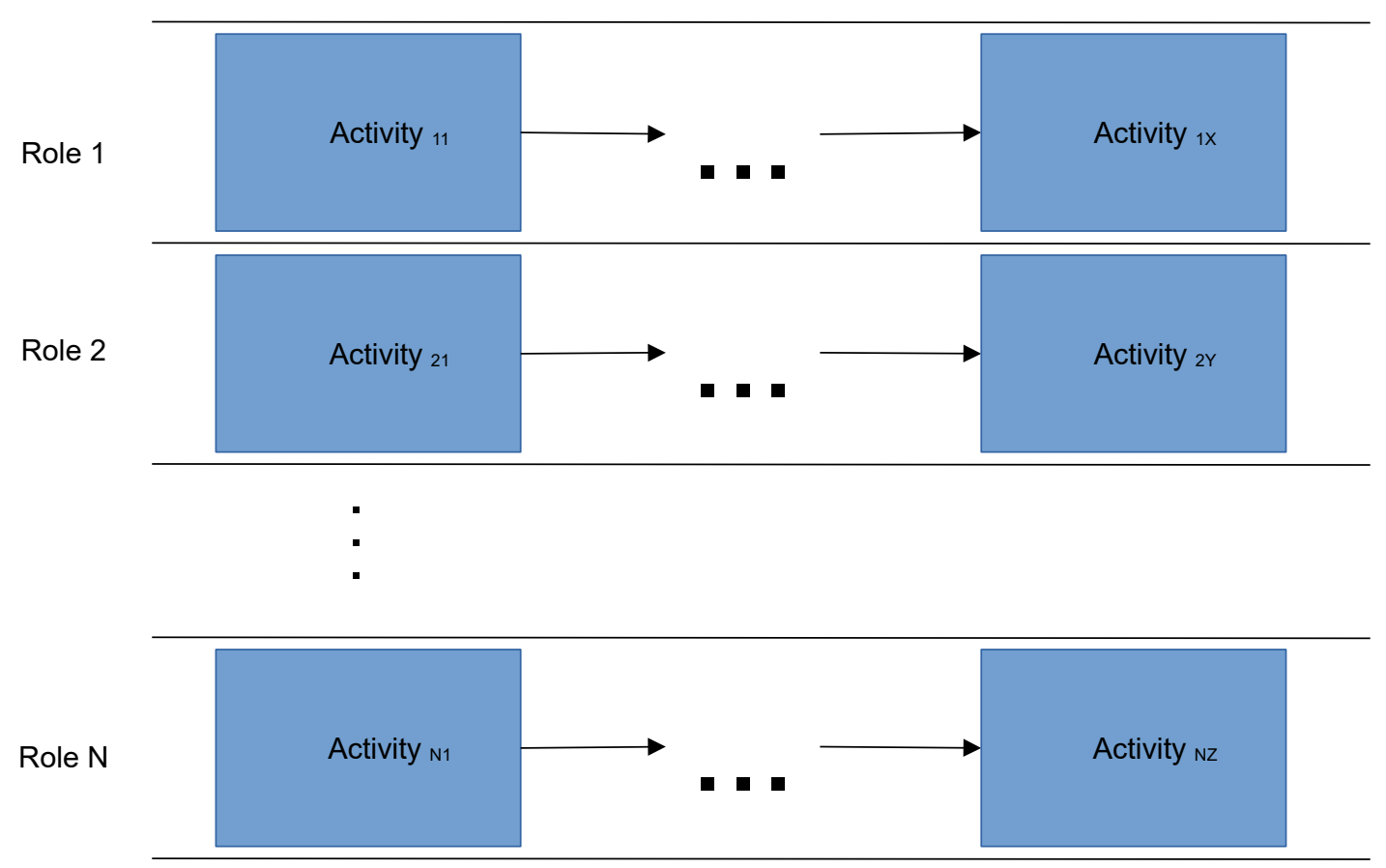

Figure 2: Multi-Role Cooperation: A given functional component in each role " $i$ " might cooperate in multiple processes " $X$ ", " $Y$ ", or " $Z$ ".

Type 1 reasoning can be used to model all pattern recognition based processes, which are essential when solutions are uncomputable, and type 2 reasoning can be used to model all logical processes which might be used when solutions are computable. Any machine learning program then can be represented as an automation of a type 1 reasoning process, and the logic of any procedural software program can be represented as an automation of a type 2 reasoning process. Therefore all programs can potentially be mapped to some path in conceptual space.

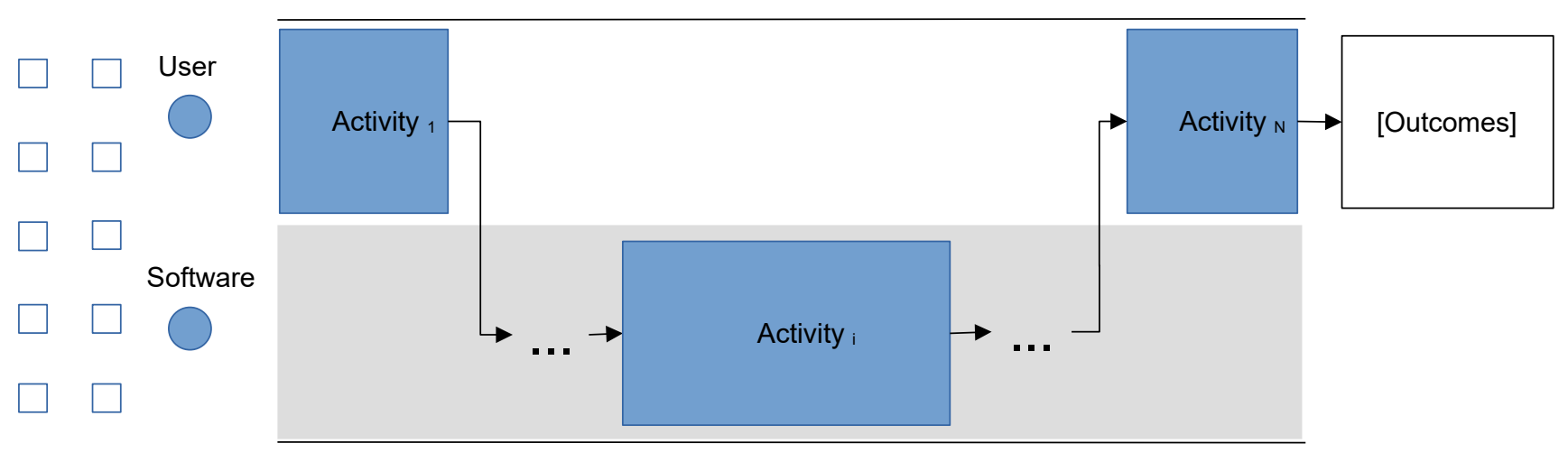

Inputs to

Process

Figure 3: Automation of process in cognitive domain can take place through hardware or software, in this case through software.

The conceptual space describes a model for processing in the cognitive domain. It is then a domain specific model. However, in order to be able to construct any computing program with such a domain specific model, we must have the capacity to construct and implement any path through conceptual 
space. In the domain of concepts, it is hypothesized that part of any logical or pattern detection based process, or any part of any algorithm automating that process that can be represented as a path through conceptual space, can be expressed as some composition of only four operations [3]. The four operations hypothesized to "span" all conceptual space are then believed to make it possible to construct any path through conceptual space. However, these operations are too abstract to be easily translated to natural language by humans without the assistance of computer automation.

\section{Scaling Execution through Cooperation}

A GCI provides the functionality to collectively execute any reasoning process, where any reasoning process is represented in terms of a path through the collective conceptual space that serves as the functional state space of GCI as a collective cognition. But GCI is just one system defined within Human-Centric Functional Modeling, which is a form of bio-mimicry that defines models for a range of human systems. One of those systems is "cooperation", which represents the inter-cellular cooperation required for organs to function. In HCFM, the domain of cognition defines the processing of information, but the domain of cooperation defines how functional components in the brain might cooperate to multiply the outcomes of that processing. The functional state space describing all possible behaviors in the domain of cooperation represents all possible modes of cooperation by which outcomes might be scaled. This functional state space describing all of these possible cooperative behaviors is the "cooperation functional state space".

One of the benefits of this HCFM approach is that all functional state spaces share properties in common, so by understanding one functional state space it is possible to understand others. The conceptual space as an open functional state space is predicted to be "spanned" by only four operations. It might then be expected that the cooperation domain might be spanned by a finite set of operations as well. Some of the operations proposed to define the cooperation domain are listed in table 1 . All of these hypothetical operations act on the domain object "activity".

Operation

Parallelize/Unparallelize

Serialize/Deserialize

Centralize/Decentralize

Distribute/Concentrate

\section{Description}

Scale processing up or down by increasing or decreasing the instances of an activity in parallel. Scale processing up or down by increasing or decreasing the instances of an activity in series. Change processing that is allowed by centralizing to increase capacity to impose restrictions, or by decentralizing to decrease capacity for restrictions to be imposed.

Change processing that is allowed by concentrating to fewer instances of an activity, or by distributing to multiple instances of an activity.

Table 1: Hypothetical operations required to span the "cooperation state space".

These operations remain to be validated. For example, any reasoning consists of a chain of logic represented by a path through the conceptual space. Any process of cooperation consists of a chain of activities in the cooperation state space. But how do we validate that we can differentiate reasoning from cooperation? That is, how do we validate that we can differentiate the process of reasoning from say the process of scaling reasoning in series through cooperation? Is reasoning confined to achieve a different outcome? Is cooperation confined to achieve the same outcome? 
If the concept of a cooperation state space is valid, and if such a set of operations does indeed span this cooperation state space, then every algorithm to distribute or parallelize computing can potentially be represented as a path in this space. As an example, an operation to parallelize an activity by increasing the number of instances of that activity to "N" might be one segment of a path through this space. An operation to then serialize that combination of parallel activities might be another segment of a path through this space.

GCI defines a functional model for computing that leverages this "cooperation functional state space" to gain the capacity to take any parallelization algorithm or any algorithm that distributes computing over multiple nodes, and to decouple that algorithm into a library of functions. For applications deployed to run on a GCI platform, that library of functions in the cooperation domain might be used to radically scale capacity to parallel-ize or distribute any computing within those applications.

Applying this cooperation domain to the logical model of hardware or software based computing process requires first defining the logical model of that process. Without such a logical model, cooperation can only be applied between computing processes as units. From reviewing a number of such computing processes that typically concern parallel or distributed computing, identifying the targeted outcome and identifying how cooperation is applied within those processes to achieve those outcomes is difficult. The cooperation is embedded too deeply into these processes to be transparent so that it can be abstracted into a separate space and independently optimized.

\begin{tabular}{|c|c|c|}
\hline Process & Targeted Outcome of Process & $\begin{array}{l}\text { Targeted Impact of } \\
\text { Parallelization and Distribution } \\
\text { on Outcome }\end{array}$ \\
\hline Offload Event Processing [5] & Unavailable & Unavailable \\
\hline $\begin{array}{l}\text { Intelligent Optimization for Bi- } \\
\text { Objective Multi-Workflow } \\
\text { Scheduling [6] }\end{array}$ & Unavailable & Unavailable \\
\hline Workflow Scheduling [7] & Unavailable & Unavailable \\
\hline Cooperative Edge Caching [8] & Unavailable & Unavailable \\
\hline \multicolumn{3}{|c|}{$\begin{array}{l}\text { Table 2: The information required to understand the path any of these algorithms take through any } \\
\text { hypothetical "cooperation state space" is unavailable and therefore can't be reused. If cooperation } \\
\text { were abstracted to a separate domain in this way, then all logical models of that cooperation, and } \\
\text { potentially instances of physical components implementing that cooperation, might be reused. }\end{array}$} \\
\hline
\end{tabular}

\section{Discussion}

Some of the problems in using GCI as a system for facilitating parallel and distributed computing include:

- How does GCI intelligently wade through all the possibilities for applying cooperation in order to scale outcomes?

- Much hardware has not yet been decoupled into a set of functional components that can interoperate through common interfaces.

- Much hardware is not open source and therefore its inner workings are unknown and cannot be modeled logically by others.

- A wide range of problems has not yet been identified using a universal model such as the conceptual space. As a result, the fitness of hardware (or software) in solving those problems can't be assessed and stored in a universal searchable index. 
Firstly, in considering whether GCI has the potential to intelligently wade through all the possibilities for applying cooperation in order to scale outcomes, a reasonable question to ask is "what actually is intelligence, and does GCI have the ability to achieve it?" Assuming that intelligence is general problem-solving ability, it is universally agreed that current AI and other computing systems, no matter how powerful, do not achieve it [9]. Whether or not GCI does have the capacity for general problemsolving ability remains to be proved, but for now we proceed on the definition of general problemsolving ability provided by Human-Centric Functional Modeling, and we proceed on the assumption that if a GCI has general problem-solving ability as satisfied by this definition, it will be intelligent. The definition of intelligence in HCFM is the ability to navigate conceptual space in a way that maintains dynamical stability in the fitness space of the cognitive system, so that cognition can reliably sustain itself until solving any problem within the abilities of the cognitive system is potentially achievable [3]. This definition applies also to GCI as a collective cognition.

Furthermore, in addition to modeling logic in some universal way, such as modeling logic as a path through conceptual space so that cooperation might be applied to it, in order to actually create hardware or software it's also important to have functional components that implement that logic in hardware or software. In order to be able to apply such a cooperation domain, that software or hardware must also have a system of interfaces which permits the required interoperation.

In addition to the lack of hardware or software implementations of functional components with such logical models, there are a lack of models of any kind. Currently, most hardware is overwhelmingly sold as black boxes without any accompanying logical model at all. In the age of GCI all of this is expected to change, allowing new hardware and software to be developed at orders of magnitude greater speed and scale [9]. Achieving a transition to incorporating collective intelligence in parallel and distributed computing however will require comprehensive metrics for a GCI to use, regarding which models of logic, which models of cooperation, and which functional components implementing those models, are more "fit" at achieving each targeted outcome. This is a massive modeling effort that has yet to be even contemplated widely, though effort to model hardware semantically do exist [11].

\section{Limitations}

A complete GCI has not yet been implemented to demonstrate general problem-solving ability across all domains. However, though this is true, a sufficient subset of GCI has been approximated in the design of various platforms to confirm the potential to exponentially increase problem-solving ability within specific domains [2], thereby confirming the value of the approach.

\section{Conclusions}

If the algorithm represented by GCI creates the potential to take any computing process that has been abstracted into a logical model (a model in which each component of logic might be implemented in a separate functional component), and to automatically search for and apply patterns of solution by which it might be possible to increase outcomes, then that is an algorithm that might be used in any other domain, such as potentially the domain of cooperation. This introduces the possibility of automatically distributing processing across multiple instances of functional components, and scaling processing by increasing the instances of those functional components in series and in parallel, as well as ensuring this effort has more value than cost to performance, so it is possible to sustain the effort at doing so, until computing outcomes are scaled as necessary to achieve a given target, or until returns from scaling diminish to the point that further investment in scaling is unproductive. By including all forms of parallel and distributed activity in such processes of cooperation, and by abstracting that cooperative activity into its own separate logical model, it might be possible to use algorithms such as GCI to facilitate parallel and distributed computing through that model. 


\section{References}

[1] Williams, A. E. Defining a Continuum from Individual, to Swarm, to Collective Intelligence, to General Collective Intelligence, International Journal of Collaborative Intelligence, in press (2022) [2] Williams, A. E. Approximating an Artificial General Intelligence or a General Collective Intelligence, International Journal of Collaborative Intelligence, in press (2022) [3] Williams A.E. (2020) A Model for Artificial General Intelligence. In: Goertzel B., Panov A., Potapov A., Yampolskiy R. (eds) Artificial General Intelligence. AGI 2020. Lecture Notes in Computer Science, vol 12177. Springer, Cham. https://doi.org/10.1007/978-3-030-52152-3_38

[4] Kahneman, Daniel. Thinking, fast and slow. Macmillan, 2011.

[5] M. Ma, J. Zhang and P. Wang, "DePo: Dynamically Offload Expensive Event Processing to the Edge of Cyber-Physical Systems" in IEEE Transactions on Parallel \& Distributed Systems, vol. 33, no. 09, pp. 2120-2132, 2022. doi: 10.1109/TPDS.2021.3135441

url: https://doi.ieeecomputersociety.org/10.1109/TPDS.2021.3135441

[6] H. Li, D. Wang, M. Zhou, Y. Fan and Y. Xia, "Multi-Swarm Co-Evolution Based Hybrid Intelligent Optimization for Bi-Objective Multi-Workflow Scheduling in the Cloud" in IEEE Transactions on Parallel \& Distributed Systems, vol. 33, no. 09, pp. 2183-2197, 2022.

doi: 10.1109/TPDS.2021.3122428

url: https://doi.ieeecomputersociety.org/10.1109/TPDS.2021.3122428

[7] X. Tang, et al.,"Cost-Efficient Workflow Scheduling Algorithm for Applications With Deadline Constraint on Heterogeneous Clouds" in IEEE Transactions on Parallel \& Distributed Systems, vol. 33, no. 09, pp. 2079-2092, 2022. doi: 10.1109/TPDS.2021.3134247, url: https://doi.ieeecomputersociety.org/10.1109/TPDS.2021.3134247

[8] X. Zhang, et al.,"Cooperative Edge Caching Based on Temporal Convolutional Networks" in IEEE Transactions on Parallel \& Distributed Systems, vol. 33, no. 09, pp. 2093-2105, 2022.

doi: 10.1109/TPDS.2021.3135257, url:

https://doi.ieeecomputersociety.org/10.1109/TPDS.2021.3135257

[9] Bishop, J. Mark. "Artificial intelligence is stupid and causal reasoning will not fix it." Frontiers in Psychology 11 (2021): 2603.

[10] Andy E. Williams, General Collective Intelligence as a Platform for Social Technology, Proceedings of the 2nd International Conference on Pervasive Computing and Social Networking [ICPCSN 2022], in print (2022).

[11] Li, Juncao, et al. "Formalizing hardware/software interface specifications." 2011 26th IEEE/ACM International Conference on Automated Software Engineering (ASE 2011). IEEE, 2011. 